Title:

\title{
Cardiac competence of the head mesoderm fades concomitant with a shift towards the head skeletal muscle programme
}

Running title:

Shifting cellular competence in the PHM

Afnan Alzamrooni, Nicoletta Murciano and Susanne Dietrich*

Institute of Biological and Biomedical Sciences

School of Pharmacy and Biomedical Sciences

University of Portsmouth

Portsmouth, UK

*Corresponding author:

Susanne Dietrich, Institute of Biological and Biomedical Sciences, School of Pharmacy and Biomedical Sciences, University of Portsmouth, St. Michael's Building, White Swan Road, Portsmouth, PO1 2DT, UK

T: +44 (0)2392 842959

F: $+44(0) 2392843565$

E: susanne.dietrich@port.ac.uk

W: http://www.port.ac.uk/institute-of-biological-and-biomedical-sciences/epigenetics-and-

developmental-biology/susanne-dietrich/Portsmouth, UK

ORCID iD: https://orcid.org/0000-0001-8688-8992 


\title{
Summary statement:
}

\author{
The head mesoderm has generic cardiac competence until head fold \\ stages. Thereafter, cardiac competence fades in the paraxial region, \\ and Bmp activates head skeletal muscle programmes instead of \\ cardiac programmes.
}

\section{Abstract}

The vertebrate head mesoderm provides the heart, the great vessels, smooth and most head skeletal muscle, and parts of the skull base. The ability to generate cardiac and smooth muscle is thought to be the evolutionary ground-state of the tissue, and initially the head mesoderm has cardiac competence throughout, even in the paraxial region that normally does not engage in cardiogenesis. How long this competence lasts, and what happens as cardiac competence fades, is not clear.

Using a wide palette of marker genes in the chicken embryo, we show that the paraxial head mesoderm has the ability to respond to Bmp, a known cardiac inducer, for a long time. However, Bmp signals are interpreted differently at different time points. Bmp triggers cardiogenesis up to early head fold stages; the ability to upregulate smooth muscle markers is retained slightly longer. Notably, as cardiac competence fades, Bmp activates the head skeletal muscle programme instead.

\section{Keywords}

Vertebrate embryos, head mesoderm, cardiogenesis, head muscles, developmental competence, Bmp.

\section{Introduction}

The vertebrate head mesoderm is the first embryonic mesoderm type to emerge during gastrulation (Camp et al., 2012; Garcia-Martinez and Schoenwolf, 1993). It delivers the heart (reviewed in (Meilhac and Buckingham, 2018; Spater et al., 2014), the great vessels (Paffett-Lugassy et al., 2013; Wang et al., 2017), the smooth muscle collar of the cardiac outflow (Waldo et al., 2005), genuine craniofacial 
and oesophageal skeletal muscles (reviewed in (Schubert et al., 2018) and parts of the skull base ((Jandzik et al., 2015); reviewed in (Kuratani, 2005)). The head mesoderm has received quite some attention since in a subpopulation of cardiogenic cells, the secondary heart field (SHF) cells, differentiation is delayed. When these cells are recruited into the heart to deliver the mature in- and outflow tract, they integrate perfectly and beat according to the rhythm set by the pre-existing cells. This is in stark contrast to cells transplanted into an adult heart to replace cells lost after a heart attack: here, cells struggle to integrate, and their autonomous beating may cause potentially fatal arrhythmias (Cui et al., 2018; Scuderi and Butcher, 2017; Spater et al., 2014).

Transcriptome analyses and grafting experiments showed that the head mesoderm is distinct from the trunk mesoderm which lacks cardiac competence and forms skeletal muscle using distinct programmes. Comparative analyses between vertebrates and invertebrate chordates indicated that this distinction is evolutionarily conserved. Moreover, since originally, chordates lacked mesodermderived cartilage and bones, cranial muscle was pharyngeal smooth muscle, and skeletal muscle was for the purpose of locomotion and formed from trunk mesoderm only, it is thought that the evolutionary default programme of the head mesoderm was to deliver cardiac and smooth muscle (Schubert et al., 2018).

Cardiogenic cells are specified but not determined as they leave the primitive streak (Lopez-Sanchez et al., 2009; Wang et al., 2013). Migration to their target area lateral of the developing neural plate is controlled by bone morphogenetic proteins (Bmp; (Song et al., 2014)). Moreover, Bmps are essential for cardiogenic cells to realise their potential and differentiate (Cui et al., 2018; Spater et al., 2014; van Wijk et al., 2007). Bmps drive cardiogenesis indirectly by suppressing the proliferation-promoting function of Fibroblast growth factors (Hutson et al., 2010; Tirosh-Finkel et al., 2010). They also control cardiogenesis directly, with Smad1/5/8-Smad4 complexes transactivating genes encoding key cardiac transcription factors such as IsI1, Nkx2.5, Gata4 and Tbx2 (Hami et al., 2011; Liberatore et al., 2002; Lien et al., 2002; Shirai et al., 2009; Si et al., 2014). Mesodermal cells expressing the pioneer factor Isl1 still have non-cardiogenic options, however the expression of $\mathrm{Nkx2.5}$ drives cells towards a cardiomyocyte fate (Gao et al., 2019; Jia et al., 2018). Nkx2.5 is not sufficient for cardiomyocyte differentiation, but the combinatorial expression of cardiogenic transcription factors allows beating cardiomyocytes to form, both in vivo and in vitro (leda et al., 2010; Luna-Zurita et al., 2016; Zhou et al., 2012).

Before and during the formation of the primitive heart, the Bmp ohnologs Bmp2 and 4 are expressed in the lateral and extraembryonic mesoderm, its overlying ectoderm and underlying pharyngeal endoderm and the neural folds (Supplementary Material S2; for Bmp phylogeny see (Hinck et al., 2016; Huminiecki et al., 2009)). Bmp2 continues to be expressed in the cardiac inflow tract, Bmp4 in the SHF 
adjacent to the heart. The heart expresses Bmp7 throughout, the ventricle expresses Bmp10. Thus, the lateral head mesoderm and the interface to the paraxial head mesoderm (PHM) is under the influence of Bmp all the time. On the other hand, the Bmp inhibitors Noggin and Chordin are expressed in Hensen's node and in the notochord in the midline of the embryo. Therefore, the PHM that remains close to the midline and dorsal to the pharynx is normally not recruited into cardiogenesis.

Using the ability of Bmps to induce cardiac markers as experimental paradigm, various studies showed that when it is first laid down, the entire head mesoderm including the PHM has cardiac competence (Andree et al., 1998; Bothe et al., 2011; Schlange et al., 2000; Schultheiss et al., 1997; Tirosh-Finkel et al., 2006; von Scheven et al., 2006a; Yamada et al., 2000). However, how long this generic cardiac competence, specifically the ability to generate cardiomyocytes, lasts, and what happens as and when cardiac competence fades, is unclear. This is because the earlier studies used different Bmps, different methods for Bmp application, different Bmp concentrations, different exposure times and different marker genes (detailed in Supplementary material S1). Thus, some studies ascribed cardiac competence to the PHM of head-fold stage embryos only, in the chicken at HH5/6 (Bothe et al., 2011; Schlange et al., 2000). Other studies suggested that the generic cardiac competence of the head mesoderm lasts until $\mathrm{HH} 10$ or even until $\mathrm{HH} 13 / 14$, an early pharyngula stage (Tirosh-Finkel et al., 2006).

To clarify this problem, and to lay the foundations for studies on the molecular regulation of cardiac competence in developing and therapeutic cardiogenic cells, we systematically investigated the ability of the PHM to respond to Bmp at different time points of development, using the chicken embryo as model. We supplied Bmp proteins on carrier beads, because Bmp concentrations produced by tissue culture cells are not known, and to avoid a possible contribution of additional factors released from the cells. We show that six-hour-exposure to beads loaded with $6.41 \mu \mathrm{M} \mathrm{Bmp}$ is sufficient to trigger robust expression changes for direct Bmp-response genes such as Noggin at all developmental stages, and responses were independent of the type of Bmp or bead used. Yet the full set of cardiac markers could only be activated at $\mathrm{HH} 5 / 6$, and only at this stage, completion of cardiomyocyte differentiation was observed upon prolonged Bmp treatment. As development proceeded and cardiac competence faded, distinct sets of marker genes were activated. These newly expressed markers suggested a shift away from cardiac/cardiomyocyte and smooth muscle programmes to the programme for craniofacial myogenesis. 


\section{Results and Discussion}

The key parameter affecting the response to Bmp treatment is the Bmp concentration.

In previous studies, different bead types, different Bmp concentrations and different Bmp paralogs were used (Supplementary material S1). We therefore explored first, which of these parameters would differentially affect cardiac induction in the PHM at stage $\mathrm{HH} 5 / 6$, the stage known for its generic cardiac competence throughout the head mesoderm. We focused on Is/1, a Bmp-dependent marker for the cardiogenic head mesoderm and underlying pharyngeal endoderm, and $T b \times 1$, a gene expressed in the pharyngeal endoderm, early caudal PHM and branchiomeric muscles, and repressed by Bmp ((Bothe and Dietrich, 2006; Bothe et al., 2011; Meireles Nogueira et al., 2015) and references therein). Results for all experiments are summarised in Supplementary material S2, n-numbers are in Supplementary material S3.

We found that at $0.192 \mu \mathrm{M}$, the concentration said to be used by (Schultheiss et al., 1997), Bmp did not upregulate $I s / 1$. At $1.28 \mu \mathrm{M}$ used by (Bothe et al., 2011), Is/1 was mildly, at $6.41 \mu \mathrm{M}$, Is/1 was robustly upregulated (Supplementary Figure SF2A-D). Is/1 upregulation was slightly more widespread with Affi-Gel "blue" beads than with heparin-coated acrylic "white" beads, possibly because heparin is able to retain the loaded protein (Ori et al., 2009). However, because there was no qualitative difference between marker gene responses, and because bead retention in the tissue was better for white beads, we continued with white beads. Beads loaded with $6.41 \mu \mathrm{M}$ of the Bmp ohnologs Bmp2 and Bmp4, the more distantly related paralog Bmp10 and the even further distant Bmp7 all upregulated Is/1 (Fig.S1E-I) and suppressed Tbx1 (Fig.S1J-N). Thus, in our 6 hours assay, all Bmp paralogs had the same effect, likely because all trigger the pSmad1/5/8 pathway (reviewed in (Hinck et al., 2016)). Consequently, the key parameter to study the duration of cardiac competence of the head mesoderm is the concentration of the Bmp, not the Bmp or bead type.

Only the HH5/6 PHM is able to activate the full set of cardiogenic transcription factors.

Next, we investigated whether cardiac markers can be activated in the PHM at stages $\mathrm{HH} 5 / 6, \mathrm{HH} 7 / 8$, $\mathrm{HH} / 10$, $\mathrm{HH} 13 / 14$, using $6.41 \mu \mathrm{M} \mathrm{Bmp2}$ on white beads in the same 6-hour assay as before. As markers, we selected $I s / 1, N k \times 2.5$ and $T b \times 2$, expressed in the cardiogenic mesoderm and underlying endoderm, and Gata4, Hand2, Tbx5, at the relevant stages largely confined to mesodermal tissues (Supplementary material S2 and references therein). All of these genes encode transcription factors that are necessary for cardiac development and, in combination, drive induced pluripotent stem cells into cardiogenesis ((leda et al., 2010; Luna-Zurita et al., 2016; Zhou et al., 2012), reviewed in (Cui et al., 2018; Spater et al., 2014). Moreover, Is/1, Nkx2.5, Gata4 and Tbx2 are direct Bmp targets (Brown et al., 2004; Chi et al., 2005; Hami et al., 2011; Liberatore et al., 2002; Lien et al., 2002; Pal and Khanna, 
2006; Shirai et al., 2009; Si et al., 2014)). At HH13/14, we also implanted Bmp beads between flank somites and the neural tube as control, reasoning that Hand2, Gata4, Tbx2, genes with widespread lateral/extraembryonic expression, would be upregulated there; as further control, we assayed for the down-regulation of somitic Myf5/MyoD expression (Berti et al., 2015; von Scheven et al., 2006a). Finally, we allowed HH13/14 embryos to develop overnight to test for possible delayed responses (Supplementary materials S2, S3, and data not shown).

Expression of $I s / 1$ was upregulated in the mesoderm and endoderm at stages HH5/6 as shown previously (Fig.1A; (Bothe et al., 2011). Yet we also obtained an upregulated expression at $\mathrm{HH} 7 / 8$ and 9/10, because we now used higher Bmp concentrations. At HH13/14, both in the 6-hour and in the overnight experiments, Is/1 expression occasionally expanded from the SHF towards the bead, similar to the results by Tirosh-Finkel (2006); however, an ectopic expression around the bead was not observed (Fig.1Di,ii). Similar to Is/1, both $N k x 2.5$ (Fig.1E-H) and Tbx2 expression (Fig.1I-L) was upregulated by Bmp at $\mathrm{HH} 5 / 6$ to HH9/10 (Fig.1E-G; I-K). At HH13/14 however, none of these markers changed expression in the head (Fig.1Hi,ii; Li,ii). In the trunk Tbx2 was upregulated, in line with its association with trunk lateral mesoderm development (Fig.1Liii). Hand2 expression was upregulated around the Bmp beads in the HH5/6 to 9/10 PHM and in the HH13/14 trunk (Fig.1M-O,Piii). In the $\mathrm{HH} 13 / 14$ head, again only an expansion of the expression domain towards the bead was observed, and only in 1 of 4 embryos (Fig.1Pi,ii). Gata4 was upregulated in the PHM at HH5/6 and 7/8 (Fig.1Q$\mathrm{R}$ ), not however at $\mathrm{HH} / 10$ or $\mathrm{HH} 13 / 14$ (Fig.1S, Ti,ii); in the trunk, ectopic expression was induced (Fig.1Tiii). Tbx5 had been described as a Bmp-unresponsive gene (Yamada et al., 2000), but we were able to obtain upregulated expression at HH5/6 (Fig.1U), possibly because we used a higher Bmp concentration compared to the previous study. Nonetheless, beyond HH5/6, Tbx5 never responded to Bmp (Fig.1V-Xiii). Taken together, the full set of marker genes used here was only ever activated at $\mathrm{HH} 5 / 6$, thereafter the set of Bmp-responsive cardiac genes became successively more limited.

\section{Only the HH5/6 PHM is able to complete cardiomyocyte differentiation}

Our data suggest that the chicken PHM may retain cardiac competence until HH5/6. Yet the head mesoderm is laid down by the primitive streak at HH3 (Camp et al., 2012; Garcia-Martinez and Schoenwolf, 1993). Thus at HH5/6, the cardiac mesoderm and the PHM had already some 10-12 hours of Bmp-dependent or Bmp-independent development, respectively. To test whether and when the PHM may be able to complete cardiomyocyte differentiation, we again implanted Bmp beads at stages $\mathrm{HH} 5 / 6$ to $\mathrm{HH} 13 / 14$, this time assaying for the expression of the Mef2c and Myocd, two transcription factors associated with the activation of genes encoding muscle structural proteins and often part of cardiomyocyte-inducing gene cocktails (reviewed in (Cui et al., 2018; Gordon, 2018; Spater et al., 2014). We also investigated expression changes of Myom1, Myh15/sarcomeric Myosins, Tnnt2, all 
indicating terminal differentiation of striated (cardiac and skeletal) muscle (see Supplementary Material S2). When embryos were exposed to Bmp for 6 hours, none of these markers was induced at any stage, even when the Bmp concentration was further increased to $19.23 \mu \mathrm{M}$ (Fig.2A-F, Supplementary Figure SF2). When the embryos were incubated overnight, then sarcomeric Myosin

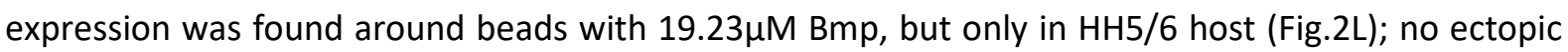
expression was ever seen at later stages (Fig.2M-R).

\section{After HH5/6 Bmp upregulates smooth muscle and skeletal muscle precursor markers}

Our data indicate that generic cardiac competence in the head mesoderm fades after HH5/6. Yet Is/1, which marks cells with myocardial but also vascular, smooth or head skeletal muscle potential (Jia et al., 2018; Nathan et al., 2008), and Hand2, a marker for head and trunk lateral mesoderm (Firulli et al., 2005; Lopez-Sanchez et al., 2009), could be activated in the PHM as late as HH10 and its expression dorsally expanded at $\mathrm{HH} 13 / 14$. This suggests that the PHM remains Bmp responsive. Moreover, Bmp is expressed in the pre-chordal plate, the pharyngeal pouches, the otic vesicle and the dorsal neural tube, suggesting that some regions of the PHM are exposed to Bmp (Bothe et al., 2011; von Scheven et al., 2006a). This then infers that, as cardiac competence fades, the PHM may respond to Bmp by activating non-(myo)cardial programmes. To test this, we first confirmed Bmp-responsiveness in the head, assaying for the expression of Noggin, a Bmp inhibitor activated in response to exogenously applied Bmp (Bothe et al., 2011; Sela-Donenfeld and Kalcheim, 2002). We also investigated the expression of Gata3, a marker for the pharyngeal endoderm and ectoderm and, in the trunk, a marker for the mesonephric duct, all sites subject to Bmp signalling (Begbie et al., 1999; Bothe and Dietrich, 2006; James and Schultheiss, 2005; Sheng and Stern, 1999). Both markers were activated within 6 hours, at all stages and sites investigated here (Fig.3A-Hiii, Supplementary Table1).

We next used $N f a t c 1$, a maker with high expression levels in the endocardium and low expression levels in blood vessels, the notochord and, at $\mathrm{HH} 13 / 14$, the pharyngeal endoderm (unpublished observations and (Wu et al., 2011)). This marker did not respond to Bmp at HH5/6 to HH9/10, suggesting that Bmp did not move cells towards an endocardial fate (Fig.3I-K). At HH13/14, however expression was upregulated in the endoderm (Fig.3Hi,ii).

Acta2 is a marker for the early cardiogenic cells, and remains expressed in embryonic cardiac (and skeletal) muscle for some time (Lopez-Sanchez et al., 2009). However, the gene has a widespread expression in the extraembryonic mesoderm, demarcating developing vascular smooth muscle (Colas et al., 2000; Lopez-Sanchez et al., 2009). Likewise, the gene is expressed in the collar of smooth muscle that demarcates the junction between the heart and the great vessels (Waldo et al., 2005), and it is smooth muscle that retains Acta2 in the adult (e.g. oesophageal smooth muscle, (Gopalakrishnan et 
al., 2015). Interestingly, Acta2 was upregulated by Bmp between at stages HH5/6 to HH9/10 (Fig.3M-

O), suggesting a prolonged competence of the PHM to produce smooth muscle.

Finally, we assayed for the expression of $M s c(M y o R)$. This gene is first activated in the rostral PHM in response to Bmp and then spreads caudally to eventually label all craniofacial skeletal muscle precursors and to participate in the activation of $M y O D$ (Bothe and Dietrich, 2006; Bothe et al., 2011; Meireles Nogueira et al., 2015; Moncaut et al., 2012; von Scheven et al., 2006b). Using a higher Bmp concentration than in our original study (Bothe et al., 2011), we were able to upregulate Msc at stages HH7/8 rather than HH9/10 (Fig.3R,S). Moreover, within the 6 hour time window, Msc was readily upregulated at HH13/14 (Fig.3Ti,ii). The response was specific to the PHM since in the trunk, Msc was not activated (Fig.3Tiii). This suggests that as cardiac competence fades, competence to engage in craniofacial myogenesis becomes established in the PHM.

\section{Conclusion}

Our study reconciles previous studies, showing that different Bmp concentrations lead to different outcomes in cardiac induction assays. Nonetheless, using a large set of marker genes, we can show that the head mesoderm has generic cardiac competence from the time of its gastrulation to early head fold stages only; smooth muscle competence is maintained slightly longer. Yet as cardiac competence ceases, Bmp signals promote the programme for head skeletal muscle formation (Fig.4). Our study allows to now depict the correct time point to analyse the cellular competence of natural and engineered cardiogenic cells. 


\section{Material and methods}

\section{Chicken embryo culture and staging}

Fertilised chicken eggs were obtained from Henry Stewart \& Co. (Lincolnshire, UK) and incubated at $38.5^{\circ} \mathrm{C}$ in a humidified incubator (LMS) to the desired stages, following the staging system of Hamburger and Hamilton (Hamburger and Hamilton, 1951). For bead implantation experiments at HH5-10, embryos were cultured on filter rings as described by Chapman and Bothe (Bothe et al., 2011; Chapman et al., 2001). Experiments at HH13/14 were performed in ovo.

\section{Recombinant proteins and bead preparation}

Recombinant Bmp proteins from R\&D Systems/Biotechne (Bmp2: 355-BM-10, Bmp4: 314-BP-10, Bmp7: 354-BP-10, Bmp10: 2926-BP-025) were reconstituted in $4 \mathrm{mM} \mathrm{HCl}$, supplemented with 0.1\% bovine serum albumin, at a concentration of $1 \mathrm{mg} / \mathrm{ml}$, and then diluted in sterile water to obtain $0.19 \mu \mathrm{M}, 1.28 \mu \mathrm{M}, 6.41 \mu \mathrm{M}$ or $19.23 \mu \mathrm{M}$. Affi-Gel blue agarose beads (BioRad) and heparin-coated acrylic beads (Sigma) were soaked in these solutions together for an hour, then washed in saline before grafting. Control beads were soaked in $0.1 \%$ BSA in saline.

\section{Microsurgical manipulation, bead implantation and embryo harvesting}

Beads were grafted into the paraxial head mesoderm (PHM) of embryos at stages $\mathrm{HH} 5 / 6, \mathrm{HH} 7 / 8$, HH9/10, HH13/14, using flame-sharpened tungsten needles ((Bothe et al., 2011; von Scheven et al., 2006a), Supplementary Figure SF1). The embryos were incubated for 6 hours, during which time they may progress to the next stage of development. Six hours of incubation allows for responses of direct and the first wave of indirect targets of signalling pathways (Bothe et al., 2011). HH5/6 and HH13/14 embryos were also incubated overnight to allow for the response of indirect targets further downstream in regulatory cascades. All embryos were harvested in 4\% PFA.

\section{Generation of templates and probes for in situ hybridisation}

Probes for in situ hybridisation and wildtype gene expression patterns are summarised in Supplementary Material S2. Templates for probe synthesis were generated using restriction endonucleases or by PCR with plasmid-based primers; antisense probes were made using the appropriate T3/T7/sp6 RNA polymerases and the DIG labelling mix (Roche/Sigma).

\section{In situ hybridisation}

In situ hybridisation followed the procedure described in (Bothe et al., 2011). Embryos were permeabilised first in methanol, then with a detergent mix (15 minutes), re-fixed and equilibrated in pre-hybridisation mix for 1 hour. Hybridisation was performed overnight at $70^{\circ} \mathrm{C}$; probes were 
detected using the alkaline phosphatase-coupled anti-DIG antibody (1:2000, Roche/Sigma) and NBT/BCIP (Roche/Sigma). After staining, embryos were fixed in 4\% PFA and stored in $80 \%$ glycerol.

\section{Immunofluorescence}

Immunofluorescence followed the procedure described in (Berti et al., 2015; Meireles Nogueira et al., 2015), using the mouse MF20 (1:500, DSHB) primary antibody and the anti-mouse $\lg G+\lg M(\mathrm{H}+\mathrm{L})-$ Alexa fluor 594 secondary antibody (1:200, Jackson Immuno).

\section{Photomicroscropy}

Images were captured on a Zeiss Axioskop with DIC and fluorescence optics, using a Zeiss AxioCam digital camera with ZEN light software. Images were processed using Adobe Photoshop 6.0.

\section{Acknowledgements}

We thank Soraya Idris-Anderson and Ashley Haywood for their assistance to the project, the IBBS section Epigenetics and Development for comments, and Frank Schubert for critically reading the manuscript. The work was funded by a UoP grant.

\section{References}

Andree, B., Duprez, D., Vorbusch, B., Arnold, H. H. and Brand, T. (1998). BMP-2 induces ectopic expression of cardiac lineage markers and interferes with somite formation in chicken embryos. Mech Dev 70, 119-131.

Begbie, J., Brunet, J. F., Rubenstein, J. L. and Graham, A. (1999). Induction of the epibranchial placodes. Development 126, 895-902.

Berti, F., Meireles Nogueira, J., Wohrle, S., Sobreira, D. R., Hawrot, K. and Dietrich, S. (2015). Time course and side-by-side analysis of mesodermal, pre-myogenic, myogenic and differentiated cell markers in the chicken model for skeletal muscle formation. J Anat 227, 361-382.

Bothe, I. and Dietrich, S. (2006). The molecular setup of the avian head mesoderm and its implication for craniofacial myogenesis. Dev Dyn 235, 2845 - 2860.

Bothe, I., Tenin, G., Oseni, A. and Dietrich, S. (2011). Dynamic control of head mesoderm patterning. Development 138, 2807-2821.

Brown, C. O., 3rd, Chi, X., Garcia-Gras, E., Shirai, M., Feng, X. H. and Schwartz, R. J. (2004). The cardiac determination factor, Nkx2-5, is activated by mutual cofactors GATA-4 and Smad1/4 via a novel upstream enhancer. J Biol Chem 279, 10659-10669.

Camp, E., Dietrich, S. and Munsterberg, A. (2012). Fate mapping identifies the origin of SHF/AHF progenitors in the chick primitive streak. PLoS One 7, e51948. 
Chapman, S. C., Collignon, J., Schoenwolf, G. C. and Lumsden, A. (2001). Improved method for chick whole-embryo culture using a filter paper carrier. Dev Dyn 220, 284-289.

Chi, X., Chatterjee, P. K., Wilson, W., 3rd, Zhang, S. X., Demayo, F. J. and Schwartz, R. J. (2005). Complex cardiac Nkx2-5 gene expression activated by noggin-sensitive enhancers followed by chamber-specific modules. Proc Natl Acad Sci U S A 102, 13490-13495.

Colas, J. F., Lawson, A. and Schoenwolf, G. C. (2000). Evidence that translation of smooth muscle alpha-actin mRNA is delayed in the chick promyocardium until fusion of the bilateral heartforming regions. Dev Dyn 218, 316-330.

Cui, M., Wang, Z., Bassel-Duby, R. and Olson, E. N. (2018). Genetic and epigenetic regulation of cardiomyocytes in development, regeneration and disease. Development 145.

Firulli, B. A., Krawchuk, D., Centonze, V. E., Vargesson, N., Virshup, D. M., Conway, S. J., Cserjesi, P., Laufer, E. and Firulli, A. B. (2005). Altered Twist1 and Hand2 dimerization is associated with Saethre-Chotzen syndrome and limb abnormalities. Nat Genet 37, 373-381.

Gao, R., Liang, X., Cheedipudi, S., Cordero, J., Jiang, X., Zhang, Q., Caputo, L., Gunther, S., Kuenne, C., Ren, Y., et al. (2019). Pioneering function of IsI1 in the epigenetic control of cardiomyocyte cell fate. Cell Res 29, 486-501.

Garcia-Martinez, V. and Schoenwolf, G. C. (1993). Primitive-streak origin of the cardiovascular system in avian embryos. Dev Biol 159, 706-719.

Gopalakrishnan, S., Comai, G., Sambasivan, R., Francou, A., Kelly, R. G. and Tajbakhsh, S. (2015). A Cranial Mesoderm Origin for Esophagus Striated Muscles. Dev Cell 34, 694-704.

Gordon, J. W. (2018). Regulation of cardiac myocyte cell death and differentiation by myocardin. Molecular and cellular biochemistry 437, 119-131.

Hamburger, V. and Hamilton, H. L. (1951). A series of normal stages in the development of the chick embryo. J. Morph. 88, 49-92.

Hami, D., Grimes, A. C., Tsai, H. J. and Kirby, M. L. (2011). Zebrafish cardiac development requires a conserved secondary heart field. Development 138, 2389-2398.

Hinck, A. P., Mueller, T. D. and Springer, T. A. (2016). Structural Biology and Evolution of the TGFbeta Family. Cold Spring Harbor perspectives in biology 8.

Huminiecki, L., Goldovsky, L., Freilich, S., Moustakas, A., Ouzounis, C. and Heldin, C. H. (2009). Emergence, development and diversification of the TGF-beta signalling pathway within the animal kingdom. BMC Evol Biol 9, 28.

Hutson, M. R., Zeng, X. L., Kim, A. J., Antoon, E., Harward, S. and Kirby, M. L. (2010). Arterial pole progenitors interpret opposing FGF/BMP signals to proliferate or differentiate. Development 137, 3001-3011.

leda, M., Fu, J. D., Delgado-Olguin, P., Vedantham, V., Hayashi, Y., Bruneau, B. G. and Srivastava, D. (2010). Direct reprogramming of fibroblasts into functional cardiomyocytes by defined factors. Cell 142, 375-386.

James, R. G. and Schultheiss, T. M. (2005). Bmp signaling promotes intermediate mesoderm gene expression in a dose-dependent, cell-autonomous and translation-dependent manner. Dev Biol 288, 113-125.

Jandzik, D., Garnett, A. T., Square, T. A., Cattell, M. V., Yu, J. K. and Medeiros, D. M. (2015). Evolution of the new vertebrate head by co-option of an ancient chordate skeletal tissue. Nature 518, 534-537.

Jia, G., Preussner, J., Chen, X., Guenther, S., Yuan, X., Yekelchyk, M., Kuenne, C., Looso, M., Zhou, Y., Teichmann, S., et al. (2018). Single cell RNA-seq and ATAC-seq analysis of cardiac progenitor cell transition states and lineage settlement. Nat Commun 9, 4877.

Kuratani, S. (2005). Craniofacial development and the evolution of the vertebrates: the old problems on a new background. Zoolog Sci 22, 1-19.

Liberatore, C. M., Searcy-Schrick, R. D., Vincent, E. B. and Yutzey, K. E. (2002). Nkx-2.5 gene induction in mice is mediated by a Smad consensus regulatory region. Dev Biol 244, 243-256. 
Lien, C. L., McAnally, J., Richardson, J. A. and Olson, E. N. (2002). Cardiac-specific activity of an Nkx2-5 enhancer requires an evolutionarily conserved Smad binding site. Dev Biol 244, 257266.

Lopez-Sanchez, C., Garcia-Masa, N., Ganan, C. M. and Garcia-Martinez, V. (2009). Movement and commitment of primitive streak precardiac cells during cardiogenesis. Int J Dev Biol 53, 14451455.

Luna-Zurita, L., Stirnimann, C. U., Glatt, S., Kaynak, B. L., Thomas, S., Baudin, F., Samee, M. A., He, D., Small, E. M., Mileikovsky, M., et al. (2016). Complex Interdependence Regulates Heterotypic Transcription Factor Distribution and Coordinates Cardiogenesis. Cell 164, 9991014.

Meilhac, S. M. and Buckingham, M. E. (2018). The deployment of cell lineages that form the mammalian heart. Nature reviews. Cardiology 15, 705-724.

Meireles Nogueira, J., Hawrot, K., Sharpe, C., Noble, A., Wood, W. M., Jorge, E. C., Goldhamer, D. J., Kardon, G. and Dietrich, S. (2015). The emergence of Pax7-expressing muscle stem cells during vertebrate head muscle development. Front Aging Neurosci 7, 62.

Moncaut, N., Cross, J. W., Siligan, C., Keith, A., Taylor, K., Rigby, P. W. and Carvajal, J. J. (2012). Musculin and TCF21 coordinate the maintenance of myogenic regulatory factor expression levels during mouse craniofacial development. Development 139, 958-967.

Nathan, E., Monovich, A., Tirosh-Finkel, L., Harrelson, Z., Rousso, T., Rinon, A., Harel, I., Evans, S. M. and Tzahor, E. (2008). The contribution of Islet1-expressing splanchnic mesoderm cells to distinct branchiomeric muscles reveals significant heterogeneity in head muscle development. Development 135, 647-657.

Ori, A., Free, P., Courty, J., Wilkinson, M. C. and Fernig, D. G. (2009). Identification of heparinbinding sites in proteins by selective labeling. Molecular \& cellular proteomics : MCP 8, 22562265.

Paffett-Lugassy, N., Singh, R., Nevis, K. R., Guner-Ataman, B., O'Loughlin, E., Jahangiri, L., Harvey, R. P., Burns, C. G. and Burns, C. E. (2013). Heart field origin of great vessel precursors relies on nkx2.5-mediated vasculogenesis. Nat Cell Biol 15, 1362-1369.

Pal, R. and Khanna, A. (2006). Role of smad- and wnt-dependent pathways in embryonic cardiac development. Stem cells and development 15, 29-39.

Schlange, T., Andree, B., Arnold, H. H. and Brand, T. (2000). BMP2 is required for early heart development during a distinct time period. Mech Dev 91, 259-270.

Schubert, F. R., Singh, A. J., Afoyalan, O., Kioussi, C. and Dietrich, S. (2018). To roll the eyes and snap a bite - function, development and evolution of craniofacial muscles. Seminars in cell \& developmental biology.

Schultheiss, T. M., Burch, J. B. and Lassar, A. B. (1997). A role for bone morphogenetic proteins in the induction of cardiac myogenesis. Genes Dev 11, 451-462.

Scuderi, G. J. and Butcher, J. (2017). Naturally Engineered Maturation of Cardiomyocytes. Frontiers in cell and developmental biology 5, 50 .

Sela-Donenfeld, D. and Kalcheim, C. (2002). Localized BMP4-noggin interactions generate the dynamic patterning of noggin expression in somites. Dev Biol 246, 311-328.

Sheng, G. and Stern, C. D. (1999). Gata2 and Gata3: novel markers for early embryonic polarity and for non-neural ectoderm in the chick embryo. Mech Dev 87, 213-216.

Shirai, M., Imanaka-Yoshida, K., Schneider, M. D., Schwartz, R. J. and Morisaki, T. (2009). T-box 2, a mediator of Bmp-Smad signaling, induced hyaluronan synthase 2 and Tgfbeta2 expression and endocardial cushion formation. Proc Natl Acad Sci U S A 106, 18604-18609.

Si, L., Shi, J., Gao, W., Zheng, M., Liu, L., Zhu, J. and Tian, J. (2014). Smad4 mediated BMP2 signal is essential for the regulation of GATA4 and Nkx2.5 by affecting the histone $\mathrm{H} 3$ acetylation in H9c2 cells. Biochem Biophys Res Commun 450, 81-86. 
Song, J., McColl, J., Camp, E., Kennerley, N., Mok, G. F., McCormick, D., Grocott, T., Wheeler, G. N. and Munsterberg, A. E. (2014). Smad1 transcription factor integrates BMP2 and Wnt3a signals in migrating cardiac progenitor cells. Proc Natl Acad Sci U S A 111, 7337-7342.

Spater, D., Hansson, E. M., Zangi, L. and Chien, K. R. (2014). How to make a cardiomyocyte. Development 141, 4418-4431.

Tirosh-Finkel, L., Elhanany, H., Rinon, A. and Tzahor, E. (2006). Mesoderm progenitor cells of common origin contribute to the head musculature and the cardiac outflow tract. Development 133, 1943-1953.

Tirosh-Finkel, L., Zeisel, A., Brodt-Ivenshitz, M., Shamai, A., Yao, Z., Seger, R., Domany, E. and Tzahor, E. (2010). BMP-mediated inhibition of FGF signaling promotes cardiomyocyte differentiation of anterior heart field progenitors. Development 137, 2989-3000.

van Wijk, B., Moorman, A. F. and van den Hoff, M. J. (2007). Role of bone morphogenetic proteins in cardiac differentiation. Cardiovasc Res 74, 244-255.

von Scheven, G., Alvares, L. E., Mootoosamy, R. C. and Dietrich, S. (2006a). Neural tube derived signals and Fgf8 act antagonistically to specify eye versus mandibular arch muscles. Development 133, 2731-2745.

von Scheven, G., Bothe, I., Ahmed, M. U., Alvares, L. E. and Dietrich, S. (2006b). Protein and genomic organisation of vertebrate $\mathrm{MyoR}$ and Capsulin genes and their expression during avian development. Gene Expr Patterns 6, 383-393.

Waldo, K. L., Hutson, M. R., Ward, C. C., Zdanowicz, M., Stadt, H. A., Kumiski, D., Abu-Issa, R. and Kirby, M. L. (2005). Secondary heart field contributes myocardium and smooth muscle to the arterial pole of the developing heart. Dev Biol 281, 78-90.

Wang, X., Chen, D., Chen, K., Jubran, A., Ramirez, A. and Astrof, S. (2017). Endothelium in the pharyngeal arches 3, 4 and 6 is derived from the second heart field. Dev Biol 421, 108-117.

Wang, X. Y., Li, Y., Ma, Z. L., Wang, L. J., Chuai, M., Munsterberg, A., Geng, J. G. and Yang, X. (2013). Retention of stem cell plasticity in avian primitive streak cells and the effects of local microenvironment. Anatomical record 296, 533-543.

Wu, B., Wang, Y., Lui, W., Langworthy, M., Tompkins, K. L., Hatzopoulos, A. K., Baldwin, H. S. and Zhou, B. (2011). Nfatc1 coordinates valve endocardial cell lineage development required for heart valve formation. Circ Res 109, 183-192.

Yamada, M., Revelli, J. P., Eichele, G., Barron, M. and Schwartz, R. J. (2000). Expression of chick Tbx-2, Tbx-3, and Tbx-5 genes during early heart development: evidence for BMP2 induction of Tbx2. Dev Biol 228, 95-105.

Zhou, L., Liu, Y., Lu, L., Lu, X. and Dixon, R. A. (2012). Cardiac gene activation analysis in mammalian non-myoblasic cells by Nkx2-5, Tbx5, Gata4 and Myocd. PLoS One 7, e48028.

\section{Figure legends}

Figure 1. Bmp activates the full set of cardiac marker at HH5/6 only.

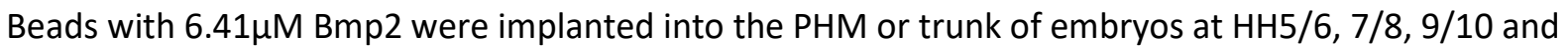
$13 / 14$, assaying for the expression of the cardiac transcription factors shown on the left. Images (i-iii) are from the same specimen. Dorsal views, rostral to the top in all except (i,ii) which are lateral views. Abbreviations: Supplementary material S3. The beads are marked by asterisks. Upregulated gene 
expression around the bead is indicated by green arrowheads, expanded expression domains by green arrows, unchanged expression by blue arrowheads. Note the declining responses to BMP after HH5/6.

\section{Figure 2. Only the HH5/6 PHM can complete myocardial differentiation.}

Implantation of control beads and beads loaded with $6.41 \mu \mathrm{M}$ or $19.23 \mu \mathrm{M} \mathrm{Bmp2}$ into the $\mathrm{HH} 5 / 6,7 / 8$, 9/10 and 13/14 PHM, followed by staining for sarcomeric Myosins. Embryos were left to develop for 6 hours (A-F, dorsal views) or overnight (G-R, ventral views). Note: ectopic cardiomyocyte

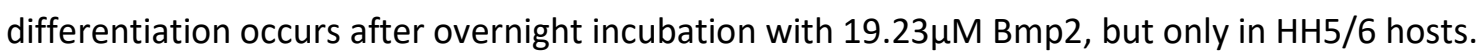

\section{Figure 3. The head skeletal muscle programme becomes available after HH5/6.}

Implantation of Bmp2 beads as in Figure 1. The upregulated expression of Noggin (A-Diii) indicates Bmp responsiveness in craniofacial tissues and the trunk. Bmp upregulated Gata3 in the pharyngeal ectoderm, endoderm and trunk mesoderm (E-Hiii) as well as Nfactc1 in the HH13/14 pharyngeal endoderm (Li,ii). (M-Pii) Acta2, a marker with sustained expression in smooth muscle, responds to Bmp at stages HH5/6 to 9/10. (Q) Msc, a marker for craniofacial skeletal muscle, is not upregulated at stage HH5/6 when the PHM has cardiac competence. Thereafter, Msc is robustly activated (R-Tii).

\section{Figure 4. Summary.}

The head mesoderm has full cardiac competence until HH5/6, and prolonged smooth muscle competence until HH9/10. In the PHM, the head skeletal muscle programme becomes available as cardiac competence declines. 
bioRxiv preprint doi: https://doi.org/10.1101/759506; this version posted September 5, 2019. The copyright holder for this preprint (which

Figure 1

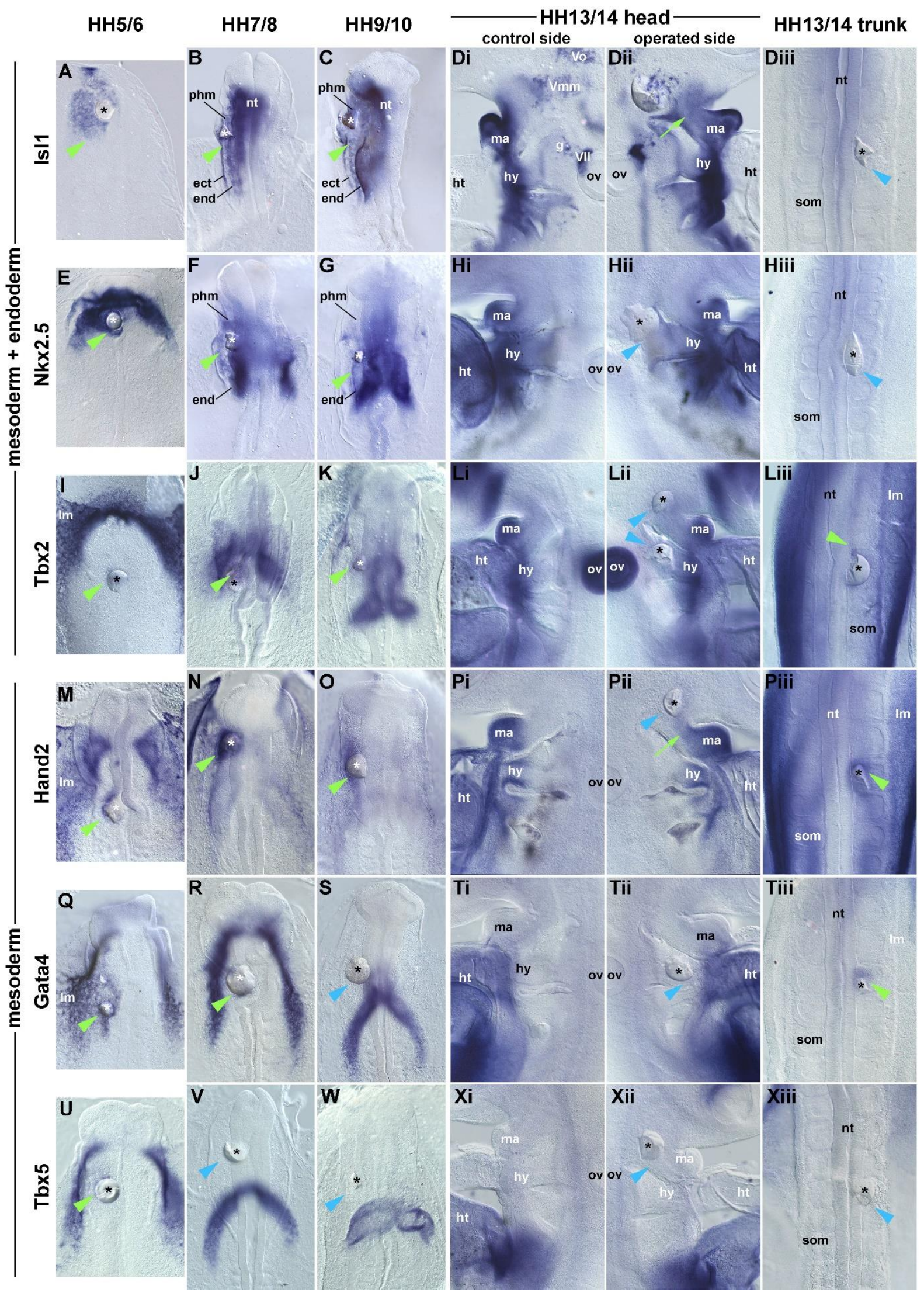


bioRxiv preprint doi: https://doi.org/10.1101/759506; this version posted September 5, 2019. The copyright holder for this preprint (which was not certified by peer review) is the author/funder, who has granted bioRxiv a license to display the preprint in perpetuity. It is made available under aCC-BY-NC-ND 4.0 International license.

Figure 2

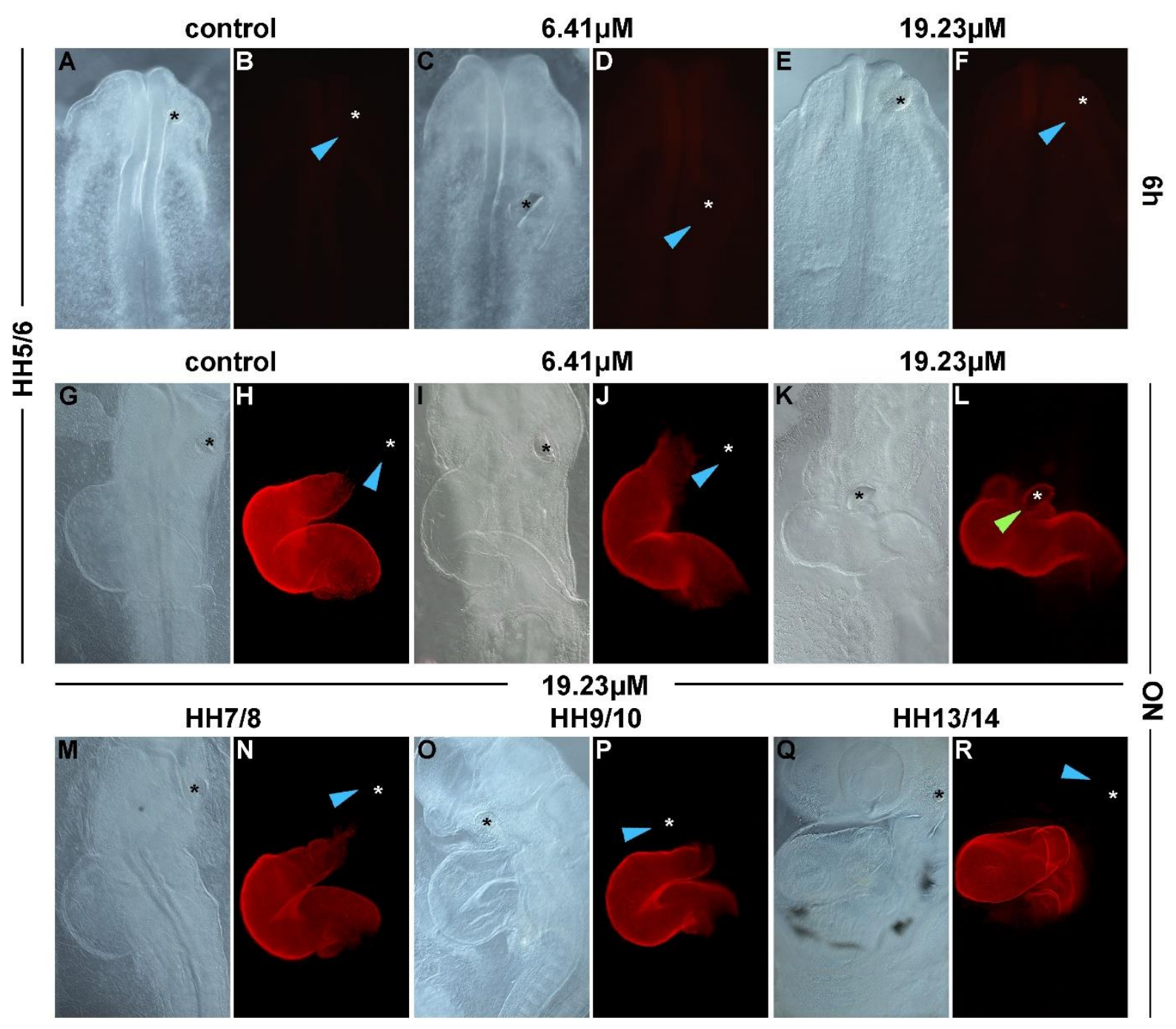


bioRxiv preprint doi: https://doi.org/10.1101/759506; this version posted September 5, 2019. The copyright holder for this preprint (which

Figure 3

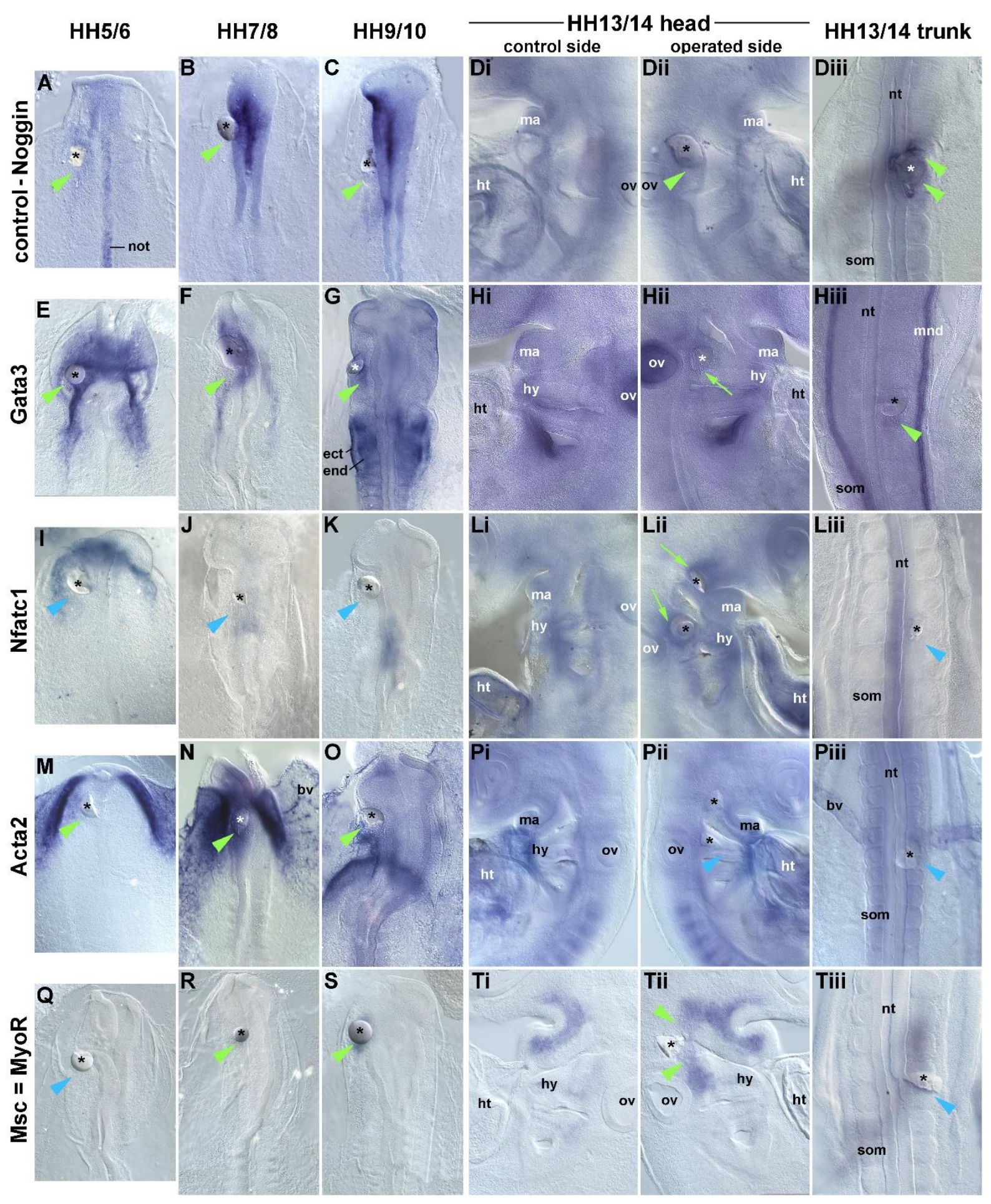


bioRxiv preprint doi: https://doi.org/10.1101/759506; this version posted September 5, 2019. The copyright holder for this preprint (which was not certified by peer review) is the author/funder, who has granted bioRxiv a license to display the preprint in perpetuity. It is made available under aCC-BY-NC-ND 4.0 International license.

\section{Figure 4}

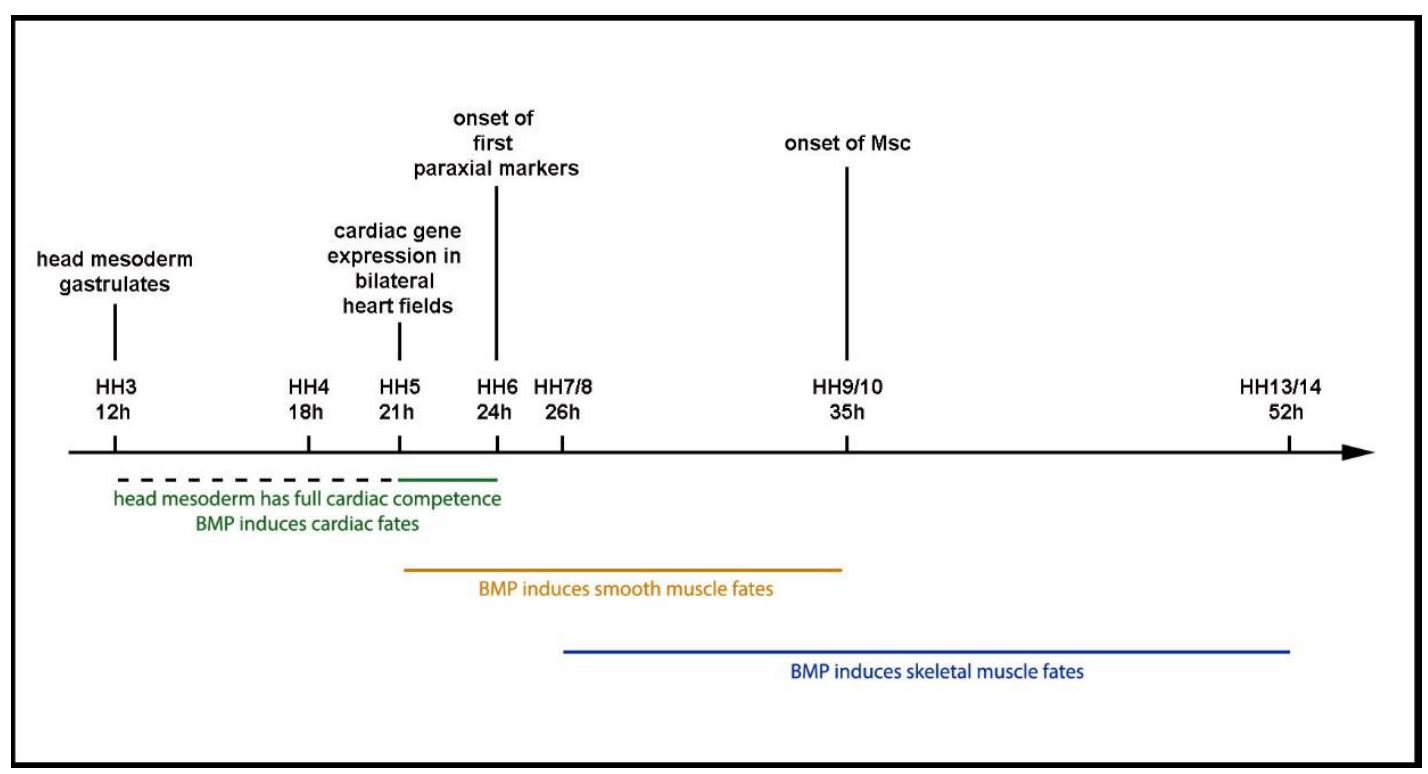

